\title{
RELATIVISTIC PARTICLES WITH TORSION IN THREE-DIMENSIONAL NON-VACUUM SPACETIMES
}

\author{
J. HERRERA, M. DE LA ROSA, AND R. M. RUBIO
}

\begin{abstract}
In this paper we analyze trajectories of spacelike curves which are critical points of a Lagrangian depending on its total torsion. We focus on two important families of spacetimes, Generalized Robertson-Walker and standard static spacetimes. For the former, we show that such trajectories are those with constant curvature. For the latter we also obtain a characterization in terms of the curvature of the trajectory, but in this case measured with an appropriate conformal metric.
\end{abstract}

\section{INTRODUCTION}

We consider a generalization of the action which models the geodesic trajectory of a relativistic particle, adding the total torsion of its worldline. In the last years, models of relativistic particles whose action contains terms depending on the curvature or torsion (or other higher curvatures) have deserved the attention of many authors. This is in part due to their interesting practical applications:

- The so-called Fermi-Bose transmutation of a charged bosonic particle in an external Chern-Simons field, to be discussed below, is connected with the appearance of a torsion term in the path integral, as is shown in [1]. This in turn may shed light on planar phenomena including superconductivity, the Hall effect and the physics of anyons.

- Curvature-depending actions can be used to describe polymer physics. This has been applied to simulate the behaviour or proteins [2].

- Some models are also linked to the Gauss-Landau-Hall problem on manifolds, which is an abstraction of the problem of determining the motion of a charged particle in a magnetic field [3].

- Their quantization provides an unusual route to the Dirac equation [4].

From the point of view of the theoretical and mathematical physics, there also exist important reasons to study these models:

- In this approach, trajectories are derived from suitable Lagrangians defined over the original spacetime, i.e. it is intrinsic, instead of requiring extra variables that provide the additional, spinning degrees of freedom.

- The Poincaré and invariance requirements show that a physically acceptable Lagrangian density must be constructed from the extrinsic curvatures of the curves in the background gravitational field.

- They follow the Polyakov argument for bosonic strings [5]. Therefore, such models furnish an extension of the usual free particle dynamics tantamount to Polyakov's expansion of the classical Nambu-Goto action for strings.

Date: April 2, 2021. 
- They allow to obtain new particle models by means of a simple and purely geometrical procedure.

The inclusion into the action of terms depending on the extrinsic curvatures of the worldline appears to be due to Pisarski [6], who extends in this way the free particle dynamical model in the same way that Polyakov extends the classical Nambu-Goto action to model the dynamic of bosonic strings [5].

Later, authors such as Nesterenko, Plyushchay and other collaborators popularized the study of these more geometric Lagrangians in the context of particle modelling [7, 8, 9, 10, 11, 12], sometimes including higher powers of the curvature or torsion [13] or force fields acting on the particles [14]. A similar analysis has been used to implement the dynamics of strings and other higher-dimensional objects [15]. It is often said that this allows to codify spinning degrees of freedom, and thus model diverse families of bosons and fermions, without having to add new, artificial coordinates lacking physical meaning. Therefore, this procedure provides an intrinsic means of representing the dynamics of relativistic particles to which the methods of modern differential geometry can be readily applied. We remark, however, that these works are developed using typically physical techniques such as Dirac's generalized Lagrangian dynamics and the corresponding Hamiltonian formalism in order to extract the trajectories from Lagrangians cotaining higher-order derivatives [16]. Most of these investigations are set in Minkowski spacetime, with the exception of e.g. [17], and usually quantize the model to some extent.

In the following decades, these variational problems have become a subject of study in the mathematical physics and pure mathematics communities, due to their aforementioned physical content and rich mathematical features. Although some have been carried out in Minkowski spacetime [18], the application of geometrical devices makes it possible to elegantly handle curved backgrounds. Indeed, the moduli space of trajectories for models whose action depends linearly on the curvature or torsion (or both) has been studied in some nontrivial gravitational fields such as spacetimes of constant curvature [19, 20]. For the class of cosmological models known as generalized Robertson-Walker spacetimes a mathematical test for the curvature-dependent action in the case of dimension three and for curves contained in the rest spaces for comoving observers has been given in [21] and a subsequent generalization to four dimensions can be found in [22]. These latter investigations, to which the present work belongs, do not address the issue of quantization.

In this work we deal with the problem of the trajectories corresponding to a functional whose Lagragian density is linear in the torsion function of the particle worldline trajectory in backgrounds covering two relevant families of spacetimes represented by warped product Lorentzian manifolds: generalized Roberson-Walker and standard static spacetimes. It is argued in $[23,24]$ that such a torsion term is relevant for the understanding of planar phenomena modelled by means of gauge field theories in two spatial dimensions, including hightemperature superconductivity and the quantum Hall effect. More precisely, this feature is produced in the effective action of charged excitations of the fields when the Lagrangian is made to contain the Chern-Simons term depending on some constant factor $\theta$. As shown in [24], for $\theta=\pi$, the torsion part leads to bosonic excitations with large momenta effectively behaving as fermions with small momenta. This occurrence is known as Fermi-Bose transmutation induced by the gauge field. More generally, for other values of the $\theta$ parameter, the excitations obey fractional statistics, i.e. they behave as anyons [25]. 
The paper is organized as follows. In Section 2, we review some generalities of the theory of variational calculus on manifolds. In particular, we deduce the field equations associated to an action which is linear in the torsion (see Theorem 2.1).

Section 3 reviews the notion of warped products and outlines some mathematical and physical properties of both generalized Robertson-Walker (GRW) and standard static spacetimes. The remaining sections focus on characterizing the solutions of the variational problem in these backgrounds. Thus, in Section 4.1, the generic equations of motion are studied in GRW spacetimes and for curves lying in spacelike slices, obtaining Theorem 4.1. This theorem states that one such curve satisfies the field equations if and only if it has constant curvature with respect to the two-dimensional geometry of the slice.

Lastly, in Section 4.2 we discuss the problem having a standard static spacetime as the background. Even if the resulting equation is not as elegant as that of the GRW scenario, we are able to ensure existence of solutions in Theorem 4.3. The study is supplemented with some numerical simulations of the trajectories corresponding to the particular case of (Anti-)de Sitter Schwarzschild spacetime.

\section{PReliminaries}

2.1. The variational problem. With such a physical scenario in mind, we will consider 3dimensional oriented and time-oriented Lorentzian manifolds $(\mathscr{M}, g)$, modelling a relativistic spacetime [26]. As discussed above, the dynamics of interest are governed by an action principle, that is to say, the allowed trajectories are the stationary curves of a functional of the form

$$
\mathscr{S}: \Gamma \longrightarrow \mathbb{R}, \quad \gamma \longmapsto \int_{\gamma} \mathscr{L} d s,
$$

where $\Gamma$ is a suitable space of curves $\gamma: I \subseteq \mathbb{R} \longrightarrow \mathscr{M}, s$ is the arc-length parameter for the Lorentzian metric $g$ and $\mathscr{L}$ is a smooth function, the so-called Lagrangian. In three dimensions, we consider the class of sufficiently differentiable timelike and spacelike curves, such that there exists an orthonormal frame $\{T, N, B\}$ along $\gamma$, where $T$ is the normalized tangent vector of the curve and whose Frenet apparatus is well defined, i.e., the following ODE are satisfied,

$$
\begin{gathered}
\nabla_{T} T=\varepsilon_{2} \kappa N \\
\nabla_{T} N=-\varepsilon_{1} \kappa T+\varepsilon_{3} \tau B \\
\nabla_{T} B=-\varepsilon_{2} \tau N .
\end{gathered}
$$

Here, $\nabla$ denotes the Levi-Civita connection on $(\mathscr{M}, g)$, and $\varepsilon_{1}, \varepsilon_{2}$ and $\varepsilon_{3}$ are the signature of the causal characters of $T, N$ and $B$, respectively. The functions $\kappa$ and $\tau$ are known as the curvature and the torsion of the curve $\gamma$. A curve satisfying this will be called a Frenet curve.

In the present paper, we will consider trajectories which are critical points of the following functional

$$
\mathscr{L}(\gamma)=\int_{\gamma} \tau d s
$$

We will consider the vast family of spacetimes admitting a splitting as a warped product (see for instance [27]). These encompass a wide variety of physically relevant models such as classical cosmological models, (Anti-)de Sitter space, models of black holes and standard static spacetimes.

Next, we present some formal developments to deal with the variational problem. The functional (4) is to be evaluated along curves belonging to some suitable space $\Gamma$ to be specified 
later. When endowed with an appropriate differentiable manifold structure, the tangent space at a curve $\gamma \in \Gamma, T_{\gamma} \Gamma$ can be naturally identified with the space of smooth vector fields $W \in \mathfrak{X}(\gamma)$ [28].

To properly make sense of whether a curve $\gamma:[a, b] \longrightarrow \mathscr{M}$ is a critical point of a functional such as (4), let us consider a variation of the curve given by a smooth two-parameter map $\Omega$ : $(-\varepsilon, \varepsilon) \times[a, b] \longrightarrow \mathscr{M}$ such that $\Omega(0, t)=\gamma(t)$. Associated to this variation are the following two vector fields

$$
V(z, t):=\frac{\partial \Omega}{\partial t}(z, t) \quad W(z, t):=\frac{\partial \Omega}{\partial z}(z, t)
$$

Clearly, $V(z, t)$ is tangent to each curve in $\Omega$, whereas $W(z, t)$ is said to be transverse. In particular, $W(t):=W(0, t) \in \mathfrak{X}(\gamma)$. As it is well known, this gives us a one-to-one correspondence between variations of $\gamma$ and elements of $T_{\gamma} \Gamma$. For this reason, the vector field $W(t)$ is known as $a$ variational vector field.

Evaluating (4) on a variation $\Omega$ with base curve $\gamma:[a, b] \longrightarrow \mathscr{M}$ gives

$$
\mathscr{L}[\Omega(z, t)]=\int_{a}^{b} \tau\left(\gamma_{z}(t)\right) \nu_{z}(t) d t
$$

where $\gamma_{z}(t):=\Omega(z, t)$ and $v_{z}(t):=\left\|\gamma_{z}^{\prime}(t)\right\|$. Then, $\gamma$ is a critical point of $\mathscr{L}$ if and only if

$$
\left.\frac{\partial}{\partial z}\right|_{z=0} \mathscr{L}[\Omega(z, t)]=0
$$

for any variation $\Omega$ constructed from $\gamma$. Note that $V=\partial \Omega / \partial t=v T$, where $T$ is the tangent unitary vector field along $\gamma_{z}$. In fact, $V(z, t)=\gamma_{z}^{\prime}(t)$. By construction, $[V, W]=0$.

2.2. Field Equations. To begin with, we will determine the general equations of motion corresponding to action (4). Let $\gamma$ be a Frenet curve in a three-dimensional spacetime and $\{T, N, B\}$, a Frenet frame, satisfying equations (1)-(3). Choosing a variation $\Omega$ with associated variational vector field $W^{1}$

$$
\frac{\partial}{\partial z} \mathscr{L}[\Omega(z, t)]=\int_{\gamma_{z}} W(\tau v) d t=\int_{\gamma_{z}}[W(\tau) v+W(v) \tau] d t
$$

where we have written $d s=v d t$. We thus need to compute $W(v), W(\tau)$. Regarding the first term and by using $v^{2}=\varepsilon_{1}\langle V, V\rangle$, we have

$$
W\left(v^{2}\right)=\varepsilon_{1} W(\langle V, V\rangle)=2 \varepsilon_{1}\left\langle\nabla_{W} V, V\right\rangle=2 \varepsilon_{1}\left\langle\nabla_{V} W, V\right\rangle=2 \varepsilon_{1} v^{2}\left\langle\nabla_{T} W, T\right\rangle
$$

where the equations $\nabla_{X} Y-\nabla_{Y} X=[X, Y]$ for any two vector fields $X, Y$ and $[V, W]=0$ have been used. Also

$$
W\left(v^{2}\right)=2 v W(v) \Longrightarrow W(v)=\varepsilon_{1} v\left\langle\nabla_{T} W, T\right\rangle
$$

Turn now to the variation of the torsion. From the Frenet equation (3), it follows that

$$
\tau=-\left\langle\nabla_{T} B, N\right\rangle
$$

Then

$$
W(\tau)=-W\left(\left\langle\nabla_{T} B, N\right\rangle\right)=-\left(\left\langle\nabla_{W} \nabla_{T} B, N\right\rangle+\left\langle\nabla_{T} B, \nabla_{W} N\right\rangle\right)
$$

\footnotetext{
${ }^{1}$ In what follows, $v$ and $\tau$ really mean $v_{z}$ and $\tau\left(\gamma_{z}\right)$, respectively.
} 
The second term is zero, since

$$
\left\langle\nabla_{T} B, \nabla_{W} N\right\rangle=-\varepsilon_{2} \tau\left\langle N, \nabla_{W} N\right\rangle=-\frac{1}{2} \varepsilon_{2} \tau W(\langle N, N\rangle)=0
$$

We rewrite the first term using the Riemann curvature by means of

$$
\nabla_{W} \nabla_{T} B=R(W, T) B+\nabla_{T} \nabla_{W} B+\nabla_{[W, T]} B
$$

The Lie bracket of $W$ and $T$ is given by

$$
[W, T]=\left[W, \frac{V}{v}\right]=-\frac{W(v)}{v^{2}} V=-\frac{W(v)}{v} T
$$

Consequently, using (3)

$$
\nabla_{[W, T]} B=-\frac{W(v)}{v} \nabla_{T} B=\frac{W(v)}{v} \varepsilon_{2} \tau N
$$

and

We also have

$$
\left\langle\nabla_{[W, T]} B, N\right\rangle=\frac{W(v)}{v} \tau=\varepsilon_{1} \tau\left\langle\nabla_{T} W, T\right\rangle
$$

$$
\left\langle\nabla_{T} \nabla_{W} B, N\right\rangle=T\left(\left\langle\nabla_{W} B, N\right\rangle\right)-\left\langle\nabla_{W} B, \nabla_{T} N\right\rangle
$$

Take the second term and apply (2)

$$
\left\langle\nabla_{W} B, \nabla_{T} N\right\rangle=\left\langle\nabla_{W} B,-\varepsilon_{1} \kappa T+\varepsilon_{3} \tau B\right\rangle
$$

Note that

$$
\left\langle\nabla_{W} B, \varepsilon_{3} \tau B\right\rangle=\frac{1}{2} \varepsilon_{3} \tau W(\langle B, B\rangle)=0
$$

and

$$
\left\langle\nabla_{W} B, T\right\rangle=W(\langle B, T\rangle)-\left\langle\nabla_{W} T, B\right\rangle=-\left\langle\nabla_{T} W, B\right\rangle-\langle[W, T], B\rangle
$$

where the last term is zero

$$
\langle[W, T], B\rangle=-\frac{W(v)}{v}\langle T, B\rangle=0
$$

Go back to the first term of (7) inside the derivative

$$
\left\langle\nabla_{W} B, N\right\rangle=\frac{\varepsilon_{2}}{\kappa}\left\langle\nabla_{W} B, \nabla_{T} T\right\rangle=\frac{\varepsilon_{2}}{\kappa}\left[W\left(\left\langle B, \nabla_{T} T\right\rangle\right)-\left\langle\nabla_{W} \nabla_{T} T, B\right\rangle\right]
$$

The first term vanishes

$$
W\left(\left\langle B, \nabla_{T} T\right\rangle\right)=\varepsilon_{2} W(\kappa\langle B, N\rangle)=0
$$

For the second term, we again insert the Riemann tensor

$$
R(W, T) T=\nabla_{W} \nabla_{T} T-\nabla_{T} \nabla_{W} T-\nabla_{[W, T]} T=\nabla_{W} \nabla_{T} T-\nabla_{T}^{2} T-\nabla_{T}[W, T]-\nabla_{[W, T]} T
$$

As $\nabla_{T}[W, T]$ and $\nabla_{[W, T]} T$ are linear combinations of $T$ and $N$, their scalar product with $B$ is zero and do not contribute. Summarizing, we can obtain

$$
W(\tau)=\varepsilon_{1} \kappa\left\langle\nabla_{T} W, B\right\rangle-\varepsilon_{1} \tau\left\langle\nabla_{T} W, T\right\rangle-\langle R(W, T) B, N\rangle+\varepsilon_{2} T\left[\frac{1}{\kappa}\left\langle\nabla_{T}^{2} W-R(W, T) T, B\right\rangle\right]
$$

Inserting results (6), (8) into the variation (5)

$$
\frac{\partial}{\partial z} \mathscr{L}[\Omega(z, t)]=\int_{\gamma_{z}}\left\{\varepsilon_{1} \kappa\left\langle\nabla_{T} W, B\right\rangle-\langle R(W, T) B, N\rangle+\varepsilon_{2} T\left[\frac{1}{\kappa}\left\langle\nabla_{T}^{2} W+R(W, T) T, B\right\rangle\right]\right\} v d t
$$


We rewrite this integral taking into account that

$$
\langle R(W, T) B, N\rangle=R(W, T, B, N)=-R(T, W, B, N)=-R(B, N, T, W)=-\langle R(B, N) T, W\rangle
$$

and the usual integration by parts

$$
\kappa\left\langle\nabla_{T} W, B\right\rangle=T[\kappa\langle W, B\rangle]-\kappa^{\prime}\langle W, B\rangle-\kappa\left\langle W, \nabla_{T} B\right\rangle
$$

Therefore

$$
\begin{aligned}
& \left.\frac{\partial}{\partial z}\right|_{z=0} \mathscr{L}[\Omega(z, t)]=\int_{\gamma} T\left[\frac{\varepsilon_{2}}{\kappa}\left\langle\nabla_{T}^{2} W+R(W, T) T, B\right\rangle+\varepsilon_{1} \kappa\langle W, B\rangle\right] d s+\int_{\gamma}[\langle R(B, N) T, W\rangle- \\
& \left.-\varepsilon_{1} \kappa^{\prime}\langle B, W\rangle-\varepsilon_{1} \kappa\left\langle\nabla_{T} B, W\right\rangle\right] d s
\end{aligned}
$$

It follows that this can be written in the standard form

$$
\left.\frac{\partial}{\partial z}\right|_{z=0} \mathscr{L}[\Omega(z, t)]=\int_{\gamma}\langle\Omega(\gamma), W\rangle d s+[\mathscr{B}(\gamma, W)]_{0}^{L}
$$

where

$$
\begin{gathered}
\Omega(\gamma)=R(B, N) T-\varepsilon_{1} \kappa^{\prime} B-\varepsilon_{1} \kappa \nabla_{T} B=R(B, N) T-\varepsilon_{1} \kappa^{\prime} B+\varepsilon_{1} \varepsilon_{2} \kappa \tau N \\
\mathscr{B}(\gamma, W)=\frac{\varepsilon_{2}}{\kappa}\left\langle\nabla_{T}^{2} W+R(W, T) T, B\right\rangle+\varepsilon_{1} \kappa\langle W, B\rangle
\end{gathered}
$$

As it is usual, we will consider an adequate space of curves ensuring that $\mathscr{B}$ vanishes (see for instance $[21,19,22])$. In particular, in this context we will consider the space of curves $\Gamma$ with fixed endpoints as well as fixed tangent and normal vectors at these. That is, for given $p, q \in \mathscr{M}, x_{1}, y_{1} \in T_{p} \mathscr{M}, x_{2}, y_{2} \in T_{q} \mathscr{M}$ with $\left\langle x_{1}, y_{1}\right\rangle=\left\langle x_{2}, y_{2}\right\rangle=0$

$$
\Gamma=\left\{\gamma:[a, b] \longrightarrow \mathscr{M}: \gamma(a)=p, \gamma(b)=q, T(a)=x_{1}, T(b)=x_{2}, N(a)=y_{1}, N(b)=y_{2}\right\}
$$

Hence, we can then enunciate the following result:

Theorem 2.1. For variations composed of curves in $\Gamma$, the boundary operator $\mathscr{B}$ corresponding to the functional (4) is zero and its Euler-Lagrange equations are given by $\Omega(\gamma)=0$, that is

$$
R(B, N) T-\varepsilon_{1} \kappa^{\prime} B+\varepsilon_{1} \varepsilon_{2} \kappa \tau N=0 .
$$

Proof. For such a variation, $W$ is zero at the endpoints and $R(W, T) T$ vanishes due to its tensorial nature. It only remains to study $\nabla_{T}^{2} W$. Begin with

$$
\nabla_{W} V=\nabla_{W}(v T)=W(v) T+v \nabla_{W} T=\nabla_{V} W=v \nabla_{T} W
$$

since $[W, V]=0$. By defining

$$
h:=\frac{W(v)}{v}=W(\log v),
$$

the first covariant derivate is

$$
\nabla_{T} W=h T+\nabla_{W} T .
$$

By taking the second derivative then

$$
\nabla_{T}^{2} W=\nabla_{T}\left(h T+\nabla_{W} T\right)=T(h) T+\varepsilon_{2} \kappa h N+\nabla_{T} \nabla_{W} T .
$$

Let us recall the expression of the Riemann tensor

$$
R(T, W) T=\nabla_{T} \nabla_{W} T-\nabla_{W} \nabla_{T} T-\nabla_{[T, W]} T .
$$


For the second term of the right, let us observe that:

$$
\nabla_{W} \nabla_{T} T=\varepsilon_{2} \nabla_{W}(\kappa N)=\varepsilon_{2} W(\kappa) N+\varepsilon_{2} \kappa \nabla_{W} N
$$

while for the third one

$$
\nabla_{[T, W]} T=h \nabla_{T} T=\varepsilon_{2} \kappa h N
$$

Therefore

$$
\nabla_{T}^{2} W=T(h) T+2 \varepsilon_{2} \kappa h N+\varepsilon_{2} W(\kappa) N+\varepsilon_{2} \kappa \nabla_{W} N .
$$

Finally, let us recall that at the endpoints, $\nabla_{W} N=0$ and the remaining terms are either multiples of $T$ or $N$. So their scalar product with $B$ cancels out, which proves that $[\mathscr{B}(\gamma, W)]_{0}^{L}=0$.

\section{SET UP}

The warped product [27] of two semi-Riemannian manifolds $\left(B, g_{B}\right)$ and $\left(F, g_{F}\right)$ (known as base and fiber respectively) with warping function $f: B \longrightarrow \mathbb{R}, f>0$ is the semi-Riemannian manifold $\left(B \times F, g^{f}\right) \equiv B \times{ }_{f} F$ where

$$
g^{f}:=\pi_{B}^{*}\left(g_{B}\right)+\left(f \circ \pi_{B}\right)^{2} \pi_{F}^{*}\left(g_{F}\right) .
$$

Here we denote by $\pi_{B}: B \times F \longrightarrow B, \pi_{F}: B \times F \longrightarrow F$ the natural projections of $B \times F$ to $B$ and $F$ respectively. Vector fields $X \in \mathfrak{X}(B)$ and $V \in \mathfrak{X}(F)$ can be lifted to vector fields $\bar{X}, \bar{V}$ in $\mathfrak{X}(B \times F)$. We will denote then as $L(B)$ and $L(F)$ the sets of lifted vector fields from $\mathfrak{X}(B)$ and $\mathfrak{X}(F)$ respectively. For simplicity, we will generally not distinguish between $f \circ \pi_{B}$ and $f$ or between $X$ and $\bar{X}$, as it will be clear from context. There exist useful formulae for evaluating the Levi-Civita connection and the Riemann curvature tensor on these fields ([27], pages 206 and 210). Indeed, given $X, Y \in L(B)$ and $V, W \in L(F)$ and denoting by $\nabla$ and $\nabla^{B}$ the Levi-Civita connections on $B \times{ }_{f} F$ and $B$, respectively, we have

$$
\begin{gathered}
\nabla_{X} Y=\nabla_{X}^{B} Y \\
\nabla_{X} V=\nabla_{V} X=\frac{X(f)}{f} V
\end{gathered}
$$

Furthermore, the following also hold ${ }^{2}$

$$
\begin{gathered}
R(V, X) Y=-\frac{H^{f}(X, Y)}{f} V \\
R(X, V) W=-\frac{g^{f}(V, W)}{f} \nabla_{X}(\nabla f)
\end{gathered}
$$

where $H^{f}$ is the Hessian of $f$.

We will focus on those such that either the base or the fibre are 1-dimensional with negative metric. First, the generalized Robertson-Walker (GRW) spacetimes are Lorentzian warped products of the type $I \times_{f} S$ where $I \subseteq \mathbb{R}$ is an open interval and $(S, g)$ is a Riemannian surface [29]. Their metric is thus

$$
g^{f}=-d t^{2}+f^{2} g
$$

\footnotetext{
${ }^{2}$ There are some sign differences between the curvature equations as given here and in [27]. This is because we take the Riemann curvature tensor to be $R(X, Y) Z=\left[\nabla_{X}, \nabla_{Y}\right] Z-\nabla_{[X, Y]} Z$, whereas in the referenced book they declare $R(X, Y) Z=\nabla_{[X, Y]} Z-\left[\nabla_{X}, \nabla_{Y}\right] Z$.
} 
with $f: I \longrightarrow \mathbb{R}$. Generalized Robertson-Walker spacetimes constitute a relevant family of relativistic models, as they cover the classical cosmological models as well as other recurring spacetimes within the field of theoretical physics, including the (Anti-)de Sitter spacetime.

Our second family of spacetimes are the well known standard static spacetimes [27]. Let us recall that these spacetimes belong to the larger family of stationary spacetimes: A spacetime is said to be stationary if there exists a globally-defined, timelike Killing vector field $U^{3}$. Let us remind that a Killing vector field $U$ is an infinitesimal isometry on $(\mathscr{M}, g)$ : i.e., the flow of $U$ determines a one-parameter family of isometries. Any member on the family of observers determined by $U$ perceives the same geometry around him as its proper time increases [26]. This is the case when the source of the gravitational field is time-independent, e.g. a Kerr black hole.

A stationary spacetime is called static if the Killing vector field $U$ is also irrotational, that is, $U$ satisfies

$$
\forall p \in \mathscr{M} \forall X, Y \in U_{p}^{\perp}:(\operatorname{curl} U)(X, Y):=g\left(\nabla_{X} U, Y\right)-g\left(\nabla_{Y} U, X\right)=0
$$

It can be argued that observers in $U$ observe no rotation in their neighbourhood (see [30] and [26] for more details). Some black hole models, like the exterior Schwarzschild solution, belong to this class.

Every static spacetime is locally a so-called standard static spacetime, which is a warped product of the form $S \times{ }_{f} I$, although the time coordinate is usually placed first. So, we will denote these spacetimes as $I_{f} \times S$ from now on. In this case, time instead of space is warped, as it is manifest in the metric

$$
g^{f}=-f^{2} d t^{2}+g
$$

where the warping function is defined on $S$.

Taking into account the decomposition of the above metrics, they admit a global foliation by spacelike slices

$$
S(t):=\{t\} \times S=\{(t, p): p \in S\}
$$

For a GRW spacetime, such slices are extrinsic spheres, that is to say, totally umbilical spacelike hypersurfaces of constant mean curvature. For a standard static spacetime, these slices are totally geodesic spacelike hypersurfaces. Our idea is then to study curves lying in slices $\gamma_{t}(s):=(t, \gamma(s))$, where $\gamma$ is a curve in $S$, as potential candidates for trajectories under the action principle (4), with the aid of equations (10)-(13). Observe that, under this restriction, we lose a degree of freedom, and so we only require to fix the tangent vectors at the endpoints. In this sense, we define the space of clamped curves $\bar{\Gamma}$ as

$$
\tilde{\Gamma}=\left\{\gamma:[a, b] \longrightarrow \mathscr{M}: \gamma(a)=p, \gamma(b)=q, T(a)=x_{1}, T(b)=x_{2}\right\}
$$

where the notation is the same as in Section 3.

\section{TRAJECTORIES IN TWO RELEVANT BACKGROUNDS}

4.1. Generalized Roberson-Walker spacetimes. Our aim in this section is to find solutions of the field equation (9) in the context of Generalized Roberson Walker spacetimes $I \times{ }_{f} S$ (with the metric defined in (14)). Consider a curve $\gamma(s)$ in $S$ with Frenet frame $\{T, N\}$. In order to write the equations of motion for curves of the form $\gamma_{t}(s)=(t, \gamma(s))$, with a fixed $t$ in the

\footnotetext{
${ }^{3}$ A vector field $U$ on a semi-Riemannian manifold is Killing if $\mathscr{L}_{X} g=0$, where $\mathscr{L}$ denotes the Lie derivative.
} 
domain of the warping function, we need to compute a Frenet frame for $\gamma_{t}(s)$, which we will denote $\left\{T^{f}, N^{f}, B^{f}\right\}$, where $T^{f}=T / f$, as well as the curvature $\kappa^{f}$ and torsion $\tau^{f}$ of $\gamma_{t}(s)$ as a function of $\kappa, f$ and their derivatives. This computation, which is based on equations (10), (11) and others, was explicitly done in [21] and we merely list the relevant results

$$
\begin{aligned}
& \nabla f=-\dot{f} \partial_{t}, \\
& \kappa^{f}=\frac{\sqrt{\varepsilon_{2}\left(\kappa^{2}-\dot{f}^{2}\right)}}{f}, \quad B^{f}=\frac{\varepsilon_{2}}{\sqrt{\varepsilon_{2}\left(\kappa^{2}-\dot{f}^{2}\right)}}\left(\dot{f} \xi+\kappa \partial_{t}\right), \\
& N^{f}=\frac{\varepsilon_{2}}{\sqrt{\varepsilon_{2}\left(\kappa^{2}-\dot{f}^{2}\right)}}\left(\kappa \xi+\dot{f} \partial_{t}\right), \quad \tau^{f}=\frac{\varepsilon_{2} \kappa^{\prime} \dot{f}}{f\left(\kappa^{2}-\dot{f}^{2}\right)},
\end{aligned}
$$

where $\varepsilon_{2}, \varepsilon_{3}$ are the signature of the causal characters of $N^{f}, B^{f}$, respectively, $\kappa$ is the curvature of $\gamma(s)$ and $\xi=N / f$. We need to compute the term $R^{f}\left(B^{f}, N^{f}\right) T^{f}$ in terms of vector fields tangent either to the base or the fiber, namely $T^{f}, \xi$ and $\partial_{t}$. To carry this out, substitute the above expressions for $N^{f}, B^{f}$ and use linearity. We obtain

$R^{f}\left(B^{f}, N^{f}\right) T^{f}=\frac{1}{\varepsilon_{2}\left(\kappa^{2}-\dot{f}^{2}\right)}\left[\kappa \dot{f} R^{f}(\xi, \xi) T^{f}+\kappa^{2} R^{f}\left(\partial_{t}, \xi\right) T^{f}+\dot{f}^{2} R^{f}\left(\xi, \partial_{t}\right) T^{f}+\dot{f} \kappa R^{f}\left(\partial_{t}, \partial_{t}\right) T^{f}\right]$.

Due to the antisymmetry of the Riemannian tensor, $R^{f}(\xi, \xi) T^{f}=R^{f}\left(\partial_{t}, \partial_{t}\right) T^{f}=0$. To compute $R^{f}\left(\partial_{t}, \xi\right) T^{f}=-R^{f}\left(\xi, \partial_{t}\right) T^{f}$, we use the warped product formula (13)

$$
R^{f}\left(\partial_{t}, \xi\right) T^{f}=-\frac{g^{f}\left(\xi, T^{f}\right)}{f} \nabla_{\partial_{t}}(\nabla f)=0
$$

concluding that

$$
R^{f}\left(B^{f}, N^{f}\right) T^{f}=0 .
$$

Hence, the field equation (9) becomes

$$
\left(\kappa^{f}\right)^{\prime} B^{f}-\varepsilon_{2} \kappa^{f} \tau^{f} N^{f}=0
$$

or, in terms of $\xi$ and $\partial_{t}$,

$$
\left(\kappa^{f}\right)^{\prime}\left(\dot{f} \xi+\kappa \partial_{t}\right)-\varepsilon_{2} \kappa^{f} \tau^{f}\left(\kappa \xi+\dot{f} \partial_{t}\right)=0 .
$$

This gives us the following two equations

$$
\left(\kappa^{f}\right)^{\prime} \dot{f}-\varepsilon_{2} \kappa^{f} \tau^{f} \kappa=0, \quad\left(\kappa^{f}\right)^{\prime} \kappa-\varepsilon_{2} \kappa^{f} \tau^{f} \dot{f}=0 .
$$

The derivative of $\kappa^{f}$ is

$$
\left(\kappa^{f}\right)^{\prime}=\frac{\varepsilon_{2} \kappa \kappa^{\prime}}{f \sqrt{\varepsilon_{2}\left(\kappa^{2}-\dot{f}^{2}\right)}}
$$

turning the first equation into

$$
\frac{\varepsilon_{2} \kappa \kappa^{\prime}}{f \sqrt{\varepsilon_{2}\left(\kappa^{2}-\dot{f}^{2}\right)}} \dot{f}-\varepsilon_{2} \frac{\sqrt{\varepsilon_{2}\left(\kappa^{2}-\dot{f}^{2}\right)}}{f} \frac{\varepsilon_{2} \kappa^{\prime} f}{f\left(\kappa^{2}-\dot{f}^{2}\right)} \kappa=0 .
$$

Note that $\kappa^{2}-\dot{f}^{2} \neq 0$. Otherwise, $N^{f}$ would be lightlike. Then, the above equation is equivalent to

$$
\dot{f} \kappa \kappa^{\prime}\left(1-\frac{\varepsilon_{2}}{f}\right)=0
$$


Next, consider the other equation of motion. Upon substitution of $\kappa^{f},\left(\kappa^{f}\right)^{\prime}, \tau^{f}$, we get

$$
\frac{\varepsilon_{2} \kappa \kappa^{\prime}}{f \sqrt{\varepsilon_{2}\left(\kappa^{2}-\dot{f}^{2}\right)}} \kappa-\varepsilon_{2} \frac{\sqrt{\varepsilon_{2}\left(\kappa^{2}-\dot{f}^{2}\right)}}{f} \frac{\varepsilon_{2} \kappa^{\prime} \dot{f}}{f\left(\kappa^{2}-\dot{f}^{2}\right)} \dot{f}=0
$$

Again using $\kappa^{2}-\dot{f}^{2} \neq 0$, the equation reduces to

$$
\frac{\kappa^{\prime}}{f}\left(\kappa^{2}-\frac{\dot{f}^{2}}{f}\right)=0
$$

Simultaneous verification of (16) and (17) occurs if and only if $\kappa^{\prime}=0$, independently of the warping function. We can therefore state:

Theorem 4.1. Let us consider $I \times_{f} S$ a three-dimensional Generalized Robertson-Walker spacetime, and let $\gamma_{t}$ be a curve with $\gamma_{t}(s)=(t, \gamma(s))$ and $t$ fixed. Then, the curve $\gamma_{t}$ is a critical point of (4) in the space of clamped curves $\tilde{\Gamma}$ if and only if $\gamma$ has constant curvature.

Remark 4.1. The fact that solutions are independent of the warping function $f$ makes it possible to find trajectories in large classes of spacetimes. This is in contrast with the case where the functional (4) is defined by using the curvature instead the torsion (see [21] for details). In these cases, the critical curves depend crucially on the warping function $f$.

In particular, curves with $\kappa^{\prime}=0$ living in Riemannian surfaces of constant curvature are well-known. Let us show some examples:

Example 4.2. Let us consider the models of 2-dimensional simply-connected, complete Riemannian manifolds with constant curvature. In this cases, the non trivial trajectories (i.e., non geodesics) are:

(i) If we assume that the fibre is the plane $\mathbb{R}^{2}$, such curves are circumferences.

(ii) If we assume that the fibre is $\mathbb{S}^{2}$, such curves are those resulting from the non empty intersection of the sphere with a (non tangent) plane which does not contain the origin.

(iii) Finally, in the case where the fibre is the hyperbolic plane, we have the circles, hypercycles and horocycles (see [31, Chapter XI]).

Observe that there are important spacetime models including these fibres, such as the Friedmann cosmological models, the static Einstein or the de Sitter spacetimes, etc.

4.2. Standard static spacetimes. Let us now consider the standard static case whose metric $g^{f}$ is given by (15). A $g^{f}$-orthonormal frame for $\gamma_{t}(s)=(t, \gamma(s))$ is $\left\{T, N, \partial_{t} / f\right\}$. Our aim, as usual, is to determine a Frenet frame $\left\{T^{f}=T, N^{f}, B^{f}\right\}$. Note that here $T, N$ are tangent to the base space whereas $\partial_{t} / f$ is tangent to the fiber. Then, applying (10)

$$
\nabla_{T^{f}}^{f} T^{f}=\nabla_{T} T=\kappa N
$$

from which it follows that $\kappa^{f}=\kappa$ and $N^{f}=N$. The only choice is then $B^{f}=\partial_{t} / f$. Let us find the torsion of $\gamma_{t}$

From (11) we get

$$
\nabla_{T^{f}}^{f} B^{f}=\nabla_{T}^{f}\left(\frac{\partial_{t}}{f}\right)=\frac{1}{f} \nabla_{T}^{f} \partial_{t}+T\left(\frac{1}{f}\right) \partial_{t}
$$

$$
\nabla_{T^{f}}^{f} B^{f}=\left[\frac{T(f)}{f^{2}}-\frac{T(f)}{f^{2}}\right] \partial_{t}=0
$$


So, as expected, these curves have $\tau^{f}=0$. Thus, the last term of (9) vanishes. We need to calculate the Riemann tensor

$$
R^{f}\left(B^{f}, N^{f}\right) T^{f}=R^{f}\left(\frac{\partial_{t}}{f}, N\right) T=-\frac{1}{f} \frac{H^{f}(N, T)}{f} \partial_{t}
$$

where in the last step we have used (12). By definition

$$
H^{f}(N, T)=H^{f}(T, N)=\left\langle\nabla_{T}(\nabla f), N\right\rangle
$$

Expand the gradient of $\nabla f$ in the orthonormal basis $\{T, N\}$

$$
\nabla f=\langle\nabla f, T\rangle T+\langle\nabla f, N\rangle N .
$$

Then

$$
\nabla_{T}(\nabla f)=T[\langle\nabla f, T\rangle] T+\langle\nabla f, T\rangle \nabla_{T} T+T[\langle\nabla f, N\rangle] N+\langle\nabla f, T\rangle \nabla_{T} N
$$

and the Hessian is

$$
H^{f}(N, T)=\kappa\langle\nabla f, T\rangle+\langle\nabla f, N\rangle^{\prime} .
$$

Therefore

$$
R^{f}\left(B^{f}, N^{f}\right) T^{f}=-\frac{1}{f^{2}}\left(\kappa\langle\nabla f, T\rangle+\langle\nabla f, N\rangle^{\prime}\right) \partial_{t}
$$

and substituting everything into the field equation (9)

$$
\frac{1}{f^{2}}\left(\kappa\langle\nabla f, T\rangle+\langle\nabla f, N\rangle^{\prime}\right) \partial_{t}+\frac{1}{f} \varepsilon_{1} \kappa^{\prime} \partial_{t}=0,
$$

or, equivalently

$$
\varepsilon_{1} \kappa^{\prime}+\frac{1}{f}\langle\nabla f, T\rangle \kappa+\frac{1}{f}\langle\nabla f, N\rangle^{\prime}=0
$$

We conclude then:

Theorem 4.2. Let $I_{f} \times S$ be a standard static spacetime, and consider a curve $\gamma_{t}(s)=(t, \gamma(s))$ for constant $t$. The curve $\gamma_{t}$ is a critical point of (4) in the space of clamped curves $\tilde{\Gamma}$ if and only if

$$
\varepsilon_{1} \kappa^{\prime}+\frac{1}{f}\langle\nabla f, T\rangle \kappa+\frac{1}{f}\langle\nabla f, N\rangle^{\prime}=0
$$

is satisfied.

We can show that previous equation (18) has always solutions in any standard static spacetime. In fact, we can prove:

Theorem 4.3. In a standard static spacetime $I_{f} \times S$, any curve $\gamma_{t}(s)=(t, \gamma(s))$ is solution of equation (18) if and only if it satisfies

$$
\tilde{\kappa}=\frac{C}{f^{2}}
$$

where $\tilde{\kappa}$ is the curvature of $\gamma$ with respect to the metric $\tilde{g}:=e^{-2 \ln f} g$ and $C$ is a constant. Hence, for each $C \in \mathbb{R}$ there exist critical points of (4) obeying (19). In particular, the geodesics of the conformal metric $\tilde{g}=e^{-2 \ln (f)} g$ satisfy the equations of motion (by considering $C=0$ ). 
Proof. For spacelike trajectories, that is, $\varepsilon_{1}=1$, (18) reads

$$
f T(\kappa)+\langle\nabla f, T\rangle \kappa=T(\langle\nabla f, N\rangle) \Longrightarrow T(f \kappa+\langle\nabla f, N\rangle)=0
$$

In other words, there is a constant of motion, $C$

$$
\langle\nabla f, N\rangle+f \kappa=C
$$

From (1) we have $\kappa=\left\langle\nabla_{T} T, N\right\rangle$. Thus

$$
\left\langle\nabla f+f \nabla_{T} T, N\right\rangle=C
$$

Alternatively, writing $\nabla \ln f=\nabla f / f$, we have

$$
C=f\left\langle\nabla \ln f+\nabla_{T} T, N\right\rangle
$$

We will now perform a conformal transformation $\tilde{g}=e^{2 \phi} g$. The corresponding Levi-Civita connections are related as follows [32]

$$
\tilde{\nabla}_{T} T=\nabla_{T} T+2 d \phi(T) T-g(T, T) \nabla \phi
$$

where, under our assumptions, $g(T, T)=1$. Taking $\phi=-\ln f$, we find

$$
g\left(\nabla \ln f+\nabla_{T} T, N\right)=g\left(\tilde{\nabla}_{T} T, N\right)
$$

Moreover, note that, for any two vectors $X, Y$

$$
\tilde{g}\left(\frac{1}{f} X, \frac{1}{f} Y\right)=\frac{1}{f^{2}} \tilde{g}(X, Y)=\frac{1}{f^{2}} e^{2 \ln f} g(X, Y)=g(X, Y)
$$

Accordingly, if $\{T, N\}$ is a Frenet frame of $\gamma$ with respect to $g$, then $\{\tilde{T}=T / f, \tilde{N}=N / f\}$ is a Frenet frame with respect to $\tilde{g}$. As a consequence

$$
g\left(\tilde{\nabla}_{T} T, N\right)=f^{2} g\left(\tilde{\nabla}_{\tilde{T}} T, \tilde{N}\right)=f^{2} g\left(\tilde{\nabla}_{\tilde{T}}(f \tilde{T}), \tilde{N}\right)=\tilde{g}\left(\tilde{\nabla}_{\tilde{T}}(f \tilde{T}), \tilde{N}\right)=f \tilde{g}\left(\tilde{\nabla}_{\tilde{T}} \tilde{T}, \tilde{N}\right)=f \tilde{\kappa}
$$

where in the last step (1) was again used. Going back to (20)

$$
C=f g\left(\tilde{\nabla}_{T} T, N\right)=f^{2} \tilde{g}\left(\tilde{\nabla}_{\tilde{T}} \tilde{T}, \tilde{N}\right)=f^{2} \tilde{\kappa} .
$$

Remark 4.3. Here we can see a major difference with the critical points of a Lagrangian depending on the curvature of the worldline. In such a case geodesics are always critical points while, in our case, this is no longer true in general. In fact, we can only ensure that geodesics for the conformal metric $\tilde{g}$ are critical points for (4).

Let us give some examples of the applicability of previous theorem. The first one will be a case where some explicit solutions are obtainable:

Example 4.4. Consider a standard static spacetime $\mathbb{R}_{f} \times H$ where

$$
H=\left\{(x, y) \in \mathbb{R}^{2}: y>0\right\}
$$

and the warping function is $f(x, y)=$ by with $b \in \mathbb{R}^{+}$. The gradient is the constant vector $\nabla f=(0, b)$. If we further admit that $\kappa=0$, that is, we restrict to straight lines, equation (18) becomes

$$
\langle\nabla f, N\rangle^{\prime}=0
$$

Since $N$ is also constant for any curve with zero curvature, the above equation holds for all straight lines, which are geodesics for both the base and the ambient. 
For the second one, we will consider the spacetime models known as the (Anti-)de Sitter Schwarzschild spacetimes. A (Anti-)de Sitter Schwarzschild model is a standard static spacetime which is both, spherically symmetric and solution of the Einstein equations for vacuum but with cosmological constant not necessarily zero.

Example 4.5. Let $(M, g)$ be a spacetime where:

$$
M=\mathbb{R} \times I \times(0,2 \pi), \quad g=-h(r) d t^{2}+h(r)^{-1} d r^{2}+r^{2} d \theta^{2},
$$

being $h(r)=f^{2}(r)=\left(1-\frac{2 M}{r}+E r^{2}\right)$ for some constants $M>0$ and $E$; and $I$ is the connected interval where $r>0$ and $f(r)>0$.

Our aim is to analyse curves $\gamma_{t}(s)=(t, \gamma(s)) \equiv(t, r(s), \theta(s))$ with constant $t$, contained in the spatial slice $\{t\} \times I \times(0,2 \pi) \equiv S$ and satisfying (19). In this case, the metric $\tilde{g}$ given in Theorem 4.3 induced on $S$ takes the form $\tilde{g}_{S}:=h(r)^{-2} d r^{2}+\frac{r^{2}}{h(r)} d \theta$.

In order to compute $\tilde{\kappa}$, we will assume that $\gamma$ is arc-length parametrized, and so, it satisfies:

$$
h(r)^{-2}(\dot{r})^{2}+\frac{r^{2}}{h(r)}(\dot{\theta})^{2}=1 .
$$

Therefore, $\tilde{\kappa}^{2}=g\left(\bar{\nabla}_{\dot{\gamma}} \dot{\gamma}, \bar{\nabla}_{\dot{\gamma}} \dot{\gamma}\right)$, where $\bar{\nabla}$ denotes the Levi-Civita connection for $\left(S, \tilde{g}_{S}\right)$. If we denote $\bar{\nabla}_{\dot{\gamma}} \dot{\gamma}=A \partial_{r}+B \partial_{\theta}$, from Koszul formulae it follows:

$$
\begin{array}{r}
A h^{-2}(r)=\dot{\gamma}\left(\dot{r} h^{-2}(r)\right)=\ddot{r} h^{-2}(r)-2 \dot{r} h^{-3}(r) h(r) \Rightarrow A=\ddot{r}-2 \dot{r} \partial_{s} \ln (h(r)), \\
B r^{2} h^{-1}(r)==\ddot{\theta} r^{2} h^{-1}(r)+2 \dot{\theta} h^{-1}(r) r \dot{r}-\dot{\theta} r^{2} h^{-2}(r) \partial_{s} h(r) \Rightarrow B=\ddot{\theta}+\dot{\theta} \partial_{s} \ln \left(\frac{r^{2}}{h(r)}\right)
\end{array}
$$

In conclusion, (19) is translated in these terms as,

$$
A^{2}+B^{2} r^{2} h(r)=C .
$$

A first nice consequence is the following result:

Proposition 4.6. The radial lines are, up to a parametrization, solutions of equation (24) for an arbitrary $C$.

Finally, we also depict numerically the trajectories in the (Anti-)de Sitter Schwarszchild spacetime for different values for the constants $M, E$ and $C$. (see Fig. 1).

\section{ACKNOWLEDGEMENTS}

The authors are grateful to the referee for his/her deep reading and making suggestions toward the improvement of this article. The first author is partially supported by the Spanish Grant MTM2016-78807-C2-2-P (MINECO and FEDER funds). The third author is partially supported by the Spanish MINECO and ERDF project Grant MTM2016-78807-C2-1-P.

\section{DATA AVAILABILITY}

The data that supports the findings of this study are available within the article. 


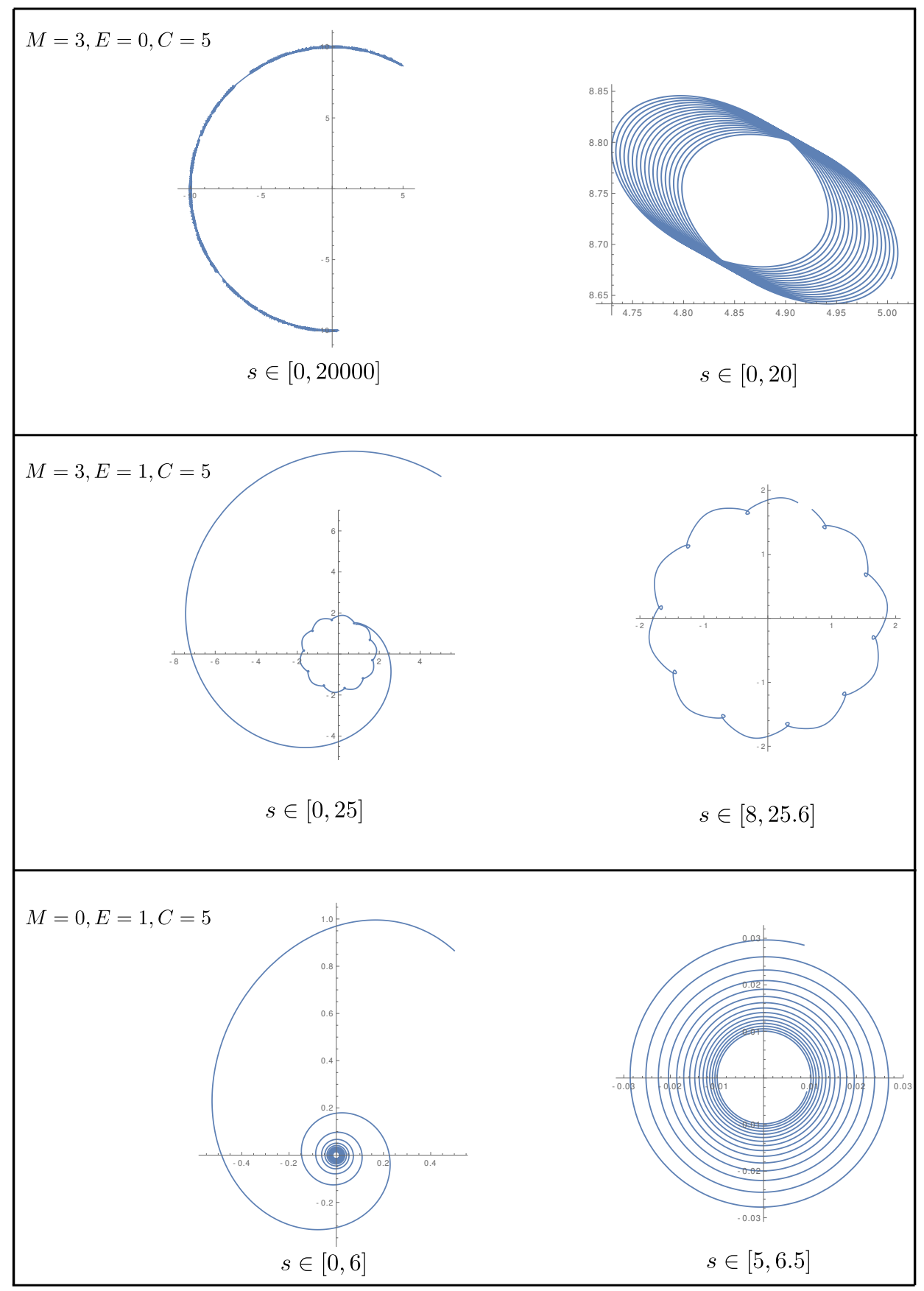

FIGURE 1. In this figure are represented different trajectories for a curve $\gamma: J \rightarrow I \times \mathbb{S}^{1}$ satisfying (21) and (24) for different values of $M, E$ and $C$. Different intervals $J$ are chosen for visualization. 


\section{REFERENCES}

[1] N. Imai, K. Ishikawa, and I. Tanaka. Quantum Theory of a Relativistic Particle with Torsion and Chern-Simons Gauge Theory. Progress of Theoretical Physics 88 (1992) 2.

[2] A. Feoli, V. V. Nesterenko, and G. Scarpetta. Functionals linear in curvature and statistics of helical proteins. Nuclear Physics B 705 [FS] (2005) 577592.

[3] M. Barros, A. Romero, J. L. Cabrerizo, and M. Fernández. The Gauss-Landau-Hall Problem on Riemannian Surfaces. Journal of Mathematical Physics 46 (2005) 112905.

[4] A. Deriglazov and A. Neressian. Rigid particle revisited: Extrinsic curvature yields the Dirac equation. Physics Letters A 378 (2014) 12241227.

[5] A. M. Polyakov. Fine Structure of Strings. Nuclear Physics B. 268 (1986) 406412.

[6] R. D. Pisarski. Field theory of paths with a curvature-dependent term. Physical Review D 34 (1986) 2.

[7] V. V. Nesterenko. Curvature and torsion of the world curve in the action of the relativistic particle. Journal of Mathematical Physics 32 (1991) 12.

[8] V. V. Nesterenko. Torsion in the action of the relativistic particle. Classical and Quantum Gravity 9 (1992) 1101-1114.

[9] V. V. Nesterenko. On a model of a relativistic particle with curvature and torsion. Journal of Mathematical Physics 34 (1993) 12.

[10] M. S. Plyushchay. Massless Point Particle with Rigidity. Modern Physics Letters A. 04 (1989) 837847.

[11] M. S. Plyushchay. Massive Relativistic Point Particle with Rigidity. International Journal of Modern Physics A. 04 (1989) 38513865.

[12] Y. A. Kuznetsov and M. S. Plyushchay. The model of the relativistic particle with curvature and torsion. Nuclear Physics B 389 (1993) 181205.

[13] V. V. Nesterenko. Dynamics of relativistic particles with Lagrangians dependent on acceleration. Journal of Mathematical Physics 36 (1995) 10.

[14] M. S. Plyushchay. Relativistic Particle with Torsion and Charged Particle in a Constant Electromagnetic Field: Identity of Evolution. Modern Physics Letters 10, 20 (1995) 1463-1469.

[15] A. L. Kholodenko and V. V. Nesterenko. Classical dynamics of the rigid string from the Willmore functional. Journal of Geometry and Physics 16 (1995) 15-26.

[16] V. V. Nesterenko. Singular Lagrangians with higher derivatives. Journal of Physics A: Mathematical and General 22 (1989) 1673-1687.

[17] V. V. Nesterenko, A. Feoli, and G. Scarpetta. Complete integrability for Lagrangians dependent on acceleration in a spacetime of constant curvature. Classical and Quantum Gravity 13 (1996) 12011211.

[18] A. Ferrández, A. Giménez, and P. Lucas. Relativistic particles and the geometry of 4-D null curves. Journal of Geometry and Physics 57 (2007) 21242135.

[19] Manuel Barros, Al Ferrández, Ma Aa Javaloyes, and Pascual Lucas. Relativistic Particles with Rigidity and Torsion in D = 3 Spacetimes. Classical and Quantum Gravity 22 (2005) 489513.

[20] A. Ferrández, J. Guerrero, M. A. Javaloyes, and P. Lucas. Particles with curvature and torsion in threedimensional pseudo-Riemannian space forms. Journal of Geometry and Physics 56 (2006) 16661687.

[21] M. Barros, M. Caballero, and M. Ortega. Massless Particles in Warped Three Spaces. International Journal of Modern Physics A, 21, 3 (2006) 461-473.

[22] M. A. Cañadas-Pinedo, M. Gutiérrez, and M. Ortega. Massless particles in generalized RobertsonWalker 4-spacetimes. Annali di Matematica 194 (2015) 259273.

[23] M. S. Plyushchay. The Model of the Relativistic Particle with Torsion. Nuclear Physics B. 362 (1991) 5472.

[24] A. M. Polyakov. Fermi-Bose Transmutations Induced by Gauge Fields. Modern Physics Letters B. 01 (1988) 455455.

[25] G. W. Semenoff. Canonical Quantum Field Theory with Exotic Statistics. Physical Review Letters 61 (1988) 517.

[26] R. K. Sachs and H. Wu. General Relativity for Mathematicians. Springer, 1st Edition (1977).

[27] B. O'Neill. Semi-Riemannian Geometry with Applications to Relativity. Academic Press, 1st Edition (1983).

[28] J. Milnor. Morse Theory. Princeton University Press, 1st Edition (1963).

[29] Luis J. Alías, Alfonso Romero, and Miguel Sánchez. Uniqueness of complete spacelike hypersurfaces of constant mean curvature in generalized Robertson-Walker spacetimes. Gen Relat Gravit 27, (1995) 7184. 
[30] Magdalena Caballero, Daniel de la Fuente, and Rafael M. Rubio. Infinitesimal relative position vector fields for observers in a reference frame and applications to conformally stationary spacetimes. Analysis and Mathematical Physics 9, 4 (2019) 1977-1990.

[31] H. Coxeter. Non-euclidean geometry. Spectrum, 1998.

[32] A. L. Besse. Einstein Manifolds. Springer, Reprint of 1st Edition (2007).

[33] Jose L. Cabrerizo, Manuel Fernandez, and Miguel Ortega. Massless particles in three-dimensional Lorentzian warped products. Journal of Mathematical Physics 48, 1 (2007) 012901.

[34] M. Sánchez. On the Geometry of Robertson-Walker Spacetimes: Geodesics. General Relativity and Gravitation, Vol. 30 (1998), No. 6.

Departamento de Matemáticas, Edificio Albert Einstein

UNIVERSIDAD DE CÓRDOBA, CAMPUS DE RABANALES,

14071 CÓRDOBA, SPAIN

Email address: jherrera@uco.es

Departamento de Matemáticas, Edificio Albert Einstein

Universidad DE CóRdoba, CAMPUS de RABANALES,

14071 CÓRDOBA, SPAIN

Departamento de Matemáticas, Edificio Albert Einstein

Universidad de Córdoba, CAMpus de RABANALES,

14071 CÓRDOBA, SPAIN 